\title{
Estudio de buenas prácticas en Clínica de Psicología. Caso Utec
}

\author{
Best Practices Study: The Psychology Clinic at UTEC
}

\author{
Edgardo R. Chacón' \\ edgardo.chacon@utec.edu.sv \\ Sandra R. de Hasbún² \\ Claudia Membreño ${ }^{3}$ \\ Profesores de la Universidad Tecnológica de El Salvador \\ Recibido: 11/05/2015 - Aceptado: 22/05/2015
}

\section{Resumen}

En este artículo se presentan los resultados de la investigación sobre las buenas prácticas realizadas en la Clínica de Psicología. El objetivo del estudio fue diseñar, a partir de su propia experiencia, un modelo de clínica psicológica que sirva de centro de prácticas, en el cual se fortalezcan las competencias de los alumnos en el área de especialidad clínica y el docente encuentre la forma de cómo realizar un proceso de enseñanza eficaz y efectivo, basado en un modelo teórico-práctico.

Se espera fortalecer la formación práctica en la labor de promoción de la salud mental; conocer la labor de proyección social universitaria, a través del servicio psicológico que se ofrece a la comunidad; evaluar el servicio de atención psicológica que brinda la Clínica de Psicología; evaluar la formación profesional de los estudiantes de la carrera de Psicología y divulgar el modelo de atención psicológica de la Clínica de Psicología.

\section{Abstract}

This article presents the results of a research conducted on the best practices of the university's psychological clinic, at the Universidad Tecnológica de El Salvador (UTEC). The aim of the study was to design, from the experience, a model of the psychological clinic to serve as a training center; here the skills of students in the area of clinical psychology can be strengthened, and teachers find a way how to perform an efficient and effective teaching process, based on a theoretical and practical model.

It is expected to strengthen the practical training in the advocacy for mental health, to communicate the university's community service work, to evaluate the psychological service offered to the community, to assess the professional training of the Psychology students, and to disseminate the model of mental care at the Psychological Clinic.

'Director de la Escuela de Psicología, Utec. Correo electrónico edgardo.chacon@utec.edu.sv 
El modelo teórico de la Clínica de Psicología de la Universidad Tecnológica de El Salvador, Utec, se ha venido transformando a través de las buenas prácticas realizadas por los docentes, que le han dado vida a la labor educativa en beneficio de los alumnos. El modelo contiene desde un enfoque pedagógico psicoanalítico hasta un contenido cognitivo conductual. El modelo de atención de la Clínica, que inició su funcionamiento en 1989, integra la proyección social hacia la comunidad, el modelo de formación pedagógico y la práctica en el área clínica realizada por los terapeutas en formación.

Esta investigación se enriquece a través de los datos obtenidos mediante la aplicación de cuestionarios y entrevistas realizadas a docentes, alumnos y pacientes de la clínica para determinar los niveles de satisfacción sobre el servicio que se les brinda en el ámbito de la formación profesional del estudiante y en la promoción y el servicio que se proporciona a los usuarios.

\section{Palabras clave}

Modelo de clínica psicológica, salud mental, buenas prácticas, satisfacción de usuarios.

La Clínica de Psicología ha expandido su área física y los horarios de atención, tomando en cuenta el incremento de los estudiantes-terapeutas de la carrera de Psicología y el aumento en la demanda del servicio por parte de la comunidad. Los estudiantes universitarios y personas particulares que requieren el servicio de atención en salud mental justifican el mantener el servicio que brinda la clínica. Asimismo, la proyección social que realiza la universidad hacia sectores de escasos recursos que lo requieren e instituciones educativas públicas que refieren sus alumnos a la clínica. Finalmente, es importante el mantener el servicio de la clínica, ya que es un centro de práctica que desarrolla en los estudiantes de la carrera competencias en el área clínica.

El problema de estudio que se planteó fue investigar las buenas prácticas del modelo de atención de la Clínica de Psicología de la Utec. Asimismo, se pretendió identificar los niveles de satisfacción y calidad en el servicio de atención psicológica que brinda la clínica a usuarios particulares y
The theoretical model of the Psychological Clinic at UTEC has been transformed through best practices by those teachers who have given life to their educational work for the benefit of students. The model contains a psychoanalytical and pedagogical approach to a cognitive behavioral content. The model of care of the clinic integrates UTEC's outreach to the community, the model of pedagogical training and the practice in the clinical area by therapists in training. The Psychological Clinic at UTEC started offering services in 1989.

This research is enriched by data obtained through questionnaires and interviews with teachers, students and patients in order to determine the levels of satisfaction towards the service they are given.

\section{Keywords}

Psychology Clinic model, mental health, best practices, customer satisfaction.

a estudiantes terapeutas en formación en la carrera de Psicología.

El nacimiento de la psicología en El Salvador se da a partir de una reseña histórica como carrera. A finales del siglo XIX se evidenciaba un atraso educativo en El Salvador, como describe Calderón de Orellana (2006), ya que no se incluían asignaturas psicológicas en la formación de maestros; sería en esa época y a inicios del siglo XX que surge la necesidad en el sistema educativo de incluir asignaturas psicopedagógicas en la formación de los docentes. Se capacitó a los maestros en las escuelas públicas y normales sobre metodologías psicopedagógicas, surgiendo el Gabinete Psicopedagógico como acuerdo oficial en septiembre de 1938, y gracias a su funcionamiento se logra la introducción de la Psicología aplicada a la educación y orientación en los procesos de enseñanza-aprendizaje.

En 1943 era llamado Gabinete Psicológico, y la Escuela Normal Superior graduaba técnicos en Psicología y en 
Educación Especial. En 1954 se había logrado la introducción de la Psicología en programas para evaluaciones psicométricas infantiles.

En El Salvador, los psicólogos Salvadora Tijerino Rizo y Alberto Walter Stahel motivaron para formar la carrera de Psicología en la Universidad de El Salvador (UES), así como el Departamento de Psicología en 1956. Los maestros pioneros del departamento de Psicología de la UES, Alberto Walter Stahel y Julio Bautista, junto con otros salvadoreños, iniciaron la formación de la carrera en Psicología.

La UES (1949) se interesa en esta materia y la incluye en su programa de formación de la Faculta de Humanidades. Los primeros cursos de verano sobre Psicología y su práctica dan frutos y logra sensibilizar a la UES; y en 1956 funda el Departamento de Psicología. La UCA brinda formación en Licenciatura en Psicología y ofrece la asistencia psicológica desde el año 1982, con el enfoque de la psicología social y comunitaria. En la Utec la profesión de Psicología da comienzo en el ciclo 01-1981, iniciando la historia de la Psicología en nuestro país desde otro enfoque, con la preparación en teoría y práctica a psicólogos en las áreas clínica y organizacional, dirigiendo las prácticas a la comunidad que no tuviera ingresos económicos 0 presupuestos para atención psicológica.

Posteriormente se amplía el servicio de la Clínica a los empleados de la universidad. Todas estas acciones logran visibilizar la importancia de la realización de prácticas psicológicas, y se reconoce que las instituciones de educación superior tienen el compromiso con la sociedad; y no se pueden desvincular de la proyección social que se encuentra entre los objetivos del Ministerio de Educación (Mined). También las prácticas en la clínica de atención psicológica permite que los estudiantes de la carrera puedan realizar servicio social, sistematizado en programas de diseño curricular aprobados por el Mined y dirigido por docentes que imparten las asignaturas relacionadas con la práctica psicológica para niños, adolescentes y adultos. Al margen de todo lo anterior, al ir creciendo la atención psicológica, este servicio permite proyectar la carrera de Psicología, ya que los alumnos obtienen competencias que les permiten adquirir identificación y ética hacia la profesión.
En El Salvador, la carrera de Psicología se inicia por Decreto Legislativo No. 955, el 28 de abril de 1988. El Órgano Judicial da a conocer reformas en el Código de Salud. Estas se hacen ante la necesidad de adaptarse a los cambios en la ciencia médica y en beneficio de asegurar la salud pública y mejorar la calidad de vida. Y lo referente a la carrera de Psicología, en el Art. 5, donde "se relacionan de un modo inmediato con la salud del pueblo las profesiones médicas, odontológicas, químico farmacéuticas, médico veterinarias, enfermería, licenciatura en Laboratorio Clínico, Psicología y otras a nivel de licenciatura, y cada una de ellas serán objeto de vigilancia por medio de un organismo legal, el en psicología, se denomina Junta de Vigilancia de la Profesión en Psicología".

Aparece el comunicado en el Diario Oficial de fecha 30 de abril de 1991, tomo 311, por orden presidencial de la República de El Salvador, decreto No. 25. El presidente Lic. Alfredo Félix Cristiani Burkard y el ministro de Salud Pública y Asistencia Social, Dr. Gilberto Lisandro Vásquez Sosa, considerando los aspectos relacionados con la salud pública y asistencia social, así como el surgimiento de la Junta de Vigilancia para la carrera de Psicología.

Es importante resaltar que la salud mental es parte inherente a la salud general de la persona; resulta imposible desligarla y abordarla como tal. La salud mental involucra el logro propio, la actitud mental positiva y la autonomía en el comportamiento que permite a los individuos identificar, confrontar y resolver sus problemas. Por tanto, la salud mental tendrá diferentes significados, dependiendo del entorno, la cultura, las influencias socioeconómicas y políticas, entre otros aspectos, por lo que su conceptualización exigirá un abordaje biológico, psicoevolutivo y relacional de la persona; y tendrá, para su total desarrollo, prerrequisitos tales como paz, educación, vivienda, alimentación, ingreso económico familiar básico, ecosistema estable, justicia social y equidad.

La salud mental ha sido definida de diversas formas; cualitativamente es más que la simple ausencia de trastornos mentales, afectaciones o discapacidades globales.

Limitándose a aspectos aislados de la persona. Es una condición dinámica y esencialmente humana que nunca se alcanzan en el sentido de un estado fijo al cual se accede 
mecánicamente. En cambio, su búsqueda y construcción incide dialécticamente en el bienestar y funcionamiento efectivo de los individuos, en su empoderamiento y desarrollo personal, en las relaciones sociales. Las condiciones de vida, la sociedad y la nación.

La salud mental, según el Comité de Salud Mental de la Organización Mundial de la Salud (OMS), se define como "el goce del grado máximo de salud que se puede lograr, es uno de los derechos fundamentales e inalienables del ser humano, sin distinción de raza, religión, ideología política o condición económica y social" (OMS, 2011).

La salud mental es un balance dinámico continuo de cada persona de sí misma y con su contexto para producir, reproducir y transformar su existencia. Incluye la idea de crisis, esperada e inesperada, común a la condición humana, siendo determinante la participación activa de la persona en la posibilidad de dar respuestas a sus propias crisis y a los cambios del contexto social. Es la posibilidad de participar en proyectos compartidos que permitan a la persona trascender en su existencia y verse reflejada en el otro, construyendo su propia identidad.

El Código de Salud prescribe, en el artículo 203: “El Ministerio, de conformidad con sus recursos y sus normas respectivas en los organismos de salud que determine, dará asistencia psicológica y psiquiátrica a los enfermos que la requieren. Esta asistencia será domiciliaria, ambulatoria o institucional y tendrá por objeto el tratamiento y control de las enfermedades y deficiencias mentales".

En la actualidad existen pocos estudios relacionados con la situación y realidad laboral del psicólogo. Morales, Díaz, Scharager, Gyslin \& Sziklai (1984) publicaron un estudio referente al campo y el papel del psicólogo en Chile. Las áreas laborales que se mencionaban en aquel tiempo eran Psicología de la salud, Psicología escolar/educacional y Psicología laboral/organizacional, y el campo académico universitario. Asimismo, en dicho estudio se menciona que, entre las distintas especialidades, la Psicología clínica abarcaba casi los dos tercios del colectivo de psicólogos, y el tercio restante se distribuía principalmente entre psicólogos escolares/educacionales y laborales/organizacionales. En la Universidad Complutense de Madrid (UCM) se desarrolló, en el 2010, una investigación con el objetivo de caracterizar el contexto clínico habitual en contraposición con el contexto de investigación. Se analizaron las características de pacientes y los tratamientos aplicados en pacientes de la Clínica Universitaria de Psicología (CUP). Se identificaron los tipos de problemas por los que demandan atención, las características de los terapeutas y su actuación terapéutica. En lo referido a las variables terapéuticas, sobre el número de sesiones de evaluación, estas oscilan entre 0 y 14 sesiones, siendo la media y la moda 4 . El $83 \%$ tuvo cuatro o menos sesiones de evaluación y solo el 3 \% tuvo más de tres sesiones. Con relación al número de sesiones de tratamiento, solo se incluyen los casos que han finalizado el tratamiento, ya fueran alta, seguimiento o evaluación postratamiento. El número de sesiones osciló entre 0 y 66 , siendo la mediana 11. El 75,3\% de los sujetos recibió 18 o menos sesiones de tratamiento (Labrador, Estupiñá \& García Vera, 2010).

Labrador, Estupiñá y García Vera (2010) han desarrollado una interesante investigación sobre la psicología clínica en España. Consideran que la psicología es percibida socialmente de forma positiva, tanto en España como en otros países. La actuación profesional de los psicólogos es considerada útil y eficaz, sus servicios se buscan y los clientes se muestran generalmente satisfechos (Berenguer y Quintanilla, 1994; Buela-Casal et al., 2005; Consumer Reports, 1995; Seligman, 1996). Aunque la información sobre cómo se realiza la práctica de la psicología clínica es escasa, en especial en el contexto clínico en contraposición con el de investigación. En España, algunos estudios señalan que los psicólogos clínicos españoles reconocen utilizar técnicas de modificación de conducta en su práctica clínica habitual, adscribiendo en su actuación profesional el modelo cognitivo-conductual. Otro grupo utiliza la modificación de conducta; y un grupo casi igual, un marco ecléctico. Sin embargo, el conocimiento preciso de la práctica asistencial de la psicología clínica es del máximo interés, pues permitiría identificar el tipo de problemas por los que se demanda atención psicológica, los tratamientos disponibles y los realmente utilizados, sus características principales (sesiones de evaluación, de tratamiento, técnicas utilizadas, etc.), y, en especial, los resultados obtenidos por cada uno de ellos (Labrador et al, 2010). 
La investigación pretendía conocer las demandas de atención y analizar los tratamientos que se llevan a cabo en una clínica de psicología de la UCM. La Clínica Universitaria de Psicología de la UCM está reconocida como Centro Sanitario por la comunidad autónoma de Madrid desde 1998. Tiene entre sus objetivos: a) prestar servicios psicológicos asistenciales de calidad; b) desarrollar la formación continuada de profesionales de Psicología Clínica y de la Salud; y c) facilitar un entorno para la investigación en psicología clínica. Para el desempeño laboral de los terapeutas cuenta, en la actualidad, con un equipo de catorce psicólogos. Todos son licenciados en Psicología con al menos un título de postgrado de nivel de magíster, que acredite su especialización en evaluación, diagnóstico y tratamiento de trastornos psicológicos, siendo el más habitual el Magister en Psicología Clínica y de la Salud de la UCM. Los psicólogos tienen una formación cognitivoconductual y entre uno y tres años de práctica clínica supervisada en el momento de incorporarse a la CUP. Sus intervenciones están supervisadas por profesionales de reconocido prestigio en Psicología clínica y de la salud o psiquiatría.

Los pacientes del CUP, como centro abierto al público en general, aceptan todo tipo de pacientes debido a la demanda de estos, no tienen criterios de exclusión. El tiempo de lista de espera es de entre uno a tres meses. La CUP recoge información sobre los pacientes con fines clínicos y de investigación, notificándoselo en el momento de iniciarse la terapia.

Labrador, Estupiñá y García Vera (2010) señalan que la distribución de diagnósticos de los pacientes, considerado de acuerdo con lo establecido según los criterios del DSM IV y DSM IV TR (APA, 1995, 2000) se destacan como los de mayor relevancia: Ios Trastornos de ansiedad (31,89\%), Sin diagnóstico (14,84\%), Trastorno del estado de ánimo (9,46 \%) y Problemas de relación (9,81\%), seguido por una serie de otros diagnósticos, en menor proporción, que hacen alrededor del $45 \%$ restante. Sobre este mismo fenómeno, Martorell y Carrasco Ortiz encontraron que en el tipo de demandas atendidas, tomando como base el DSM IV-TR (APA, 2010), se observan los diagnósticos realizados en el Servicio de Psicología Aplicada (SAP) de la Facultad de Psicología de la Universidad Nacional de Educación a Distancia (Uned).
Mayoritariamente, las principales demandas son por los trastornos del estado de ánimo (10,9\%) y los de ansiedad (31,3\%). Se destacan otros problemas de atención clínica, entre los que cabe mencionar problemas de relación paterno-filiales y conyugales $(24,7 \%)$.

La mayor parte de los pacientes son mujeres, con edades por encima de los 18 años (51,8\%). En menor medida, acuden niños de ambos sexos. Aproximadamente, entre 80 y $85 \%$ de los usuarios son adultos, y alrededor del $20 \%$ son niños; de los usuarios $23,57 \%$, pertenecen a la comunidad universitaria de la Uned. $13 \%$ son trabajadores o familiares de estos, y alrededor del $60 \%$ es personal externo.

Según Martorrell y Carrasco Ortiz (2009), el SPA ha llevado estadísticas sistematizadas de los últimos ocho años sobre la actividad del Servicio de Atención Psicológica. Se ha atendido a un total aproximado de 627 usuarios. La media de clientes atendidos por año ha sido de 78.3. En la mayoría de los casos los terapeutas atienden a cada usuario una vez por semana. El tiempo de intervención es variable, así como el número de altas, las cuales se dan una vez alcanzados los objetivos terapéuticos. Gran parte de los usuarios no llegan a finalizar su proceso al interrumpir la terapia cuando alcanzan parcialmente los objetivos trazados o experimentan una notable mejoría respecto a su sintomatología inicial o su motivo de consulta. De igual forma, es considerable el número de bajas o abandonos de la terapia. No obstante, es elevada la actividad profesional que desarrollan los terapeutas, lo cual contribuye en su formación pedagógica y se logran los objetivos de proyección social de la universidad hacia la sociedad (Martorell, Carrasco y Ortiz, 2009).

Labrador, Estupiñá y García Vera (2010), en su estudio de la CUP de la UCM, como centro sanitario por la comunidad autónoma de Madrid desde 1998, incluyó en su estudio todos los datos disponibles desde el inicio de la CUP, de junio de 1999 a febrero de 2008. El total de pacientes de la base de datos fue de 1.325 usuarios.

Labrador, Estupiñá y García Vera (2010) encontraron que el 52,45\% había obtenido alta terapéutica, el 4,21 \% baja terapéutica y $24,3 \%$ había abandonado el tratamiento. El 83 \% tuvo cuatro o menos sesiones de evaluación, y el 
$75,3 \%$ de los sujetos recibió dieciocho o menos sesiones de tratamiento. El $24,3 \%$ de los pacientes abandonó el tratamiento. El $75,5 \%$ de los pacientes recibió trece sesiones de terapia o menos. Un $68,34 \%$ había finalizado el tratamiento como altas y $31,66 \%$ lo abandonaron.

La persona que solicita consulta y terapia psicológica, cualquiera que sea el motivo de sus síntomas, se encuentra en un estado desalentador que puede ser caracterizado algunas veces por pérdida de la autoestima, sentimientos de incompetencia, desvalimiento y desesperanza. Esa sensación que desvaloriza subjetivamente deja al individuo incapaz para afrontar las demandas del entorno, y con frecuencia lo lleva a confundir el significado de los síntomas; a exagerar su severidad; a temer volverse loco, perder la razón, el trabajo, el matrimonio, entre otros aspectos.

La diversidad de síntomas-problemas que presenta el paciente va a influir en el enfoque y permitirá la orientación del modelo que se adopte en el tratamiento por parte del terapeuta. Este tratamiento, en la mayoría de ocasiones, es eficaz, pero en algunos casos puede que no.

Algunos modelos actuales de psicoterapia basados en criterios de APA, según División 12, Arturo Bados López (2002) y Amparo Belloch (1995), son los siguientes: psicodinámicos, humanísticos-existenciales, conductuales, sistémicos, cognitivos y de competencias psicoterapéuticas (Ruiz Sánchez et al, 2013).

El modelo cognitivo se remonta a diferentes teorías. Por ejemplo, Lungwitz (1926) propone un modelo de terapia bastante cercano a la terapia cognitiva conductual; $\mathrm{K}$. Horney (1950) desarrolla su teoría referente a los actitudes neuróticas; A. Adler (1892-1937) da a conocer su modelo cognitivo de la psicopatología y la psicoterapia, por lo que lo llaman el fundador de las terapias cognitivas.

La terapia cognitiva se entiende como un procedimiento activo, directivo, estructurado y de tiempo limitado, que se utiliza para tratar distintas alternaciones psiquiátricas (por ejemplo, la depresión, la ansiedad, las fobias, los problemas relacionados con el dolor, entre otras). Se basa en el supuesto teórico subyacente de que los efectos y la conducta de un individuo están determinados en gran medida por el modo que este tiene de estructurar el mundo (Beck, 1967 y 1976).

Beck dedicó parte de su trabajo al estudio de la depresión. observó que en este y otros trastornos emocionales las estructuras de las experiencias de los individuos determinan sus sentimientos y conducta. A este concepto de estructura cognitiva se le llama esquema cognitivo, y en el ámbito clínico, supuestos personales (Beck, 1979).

Básicamente los conceptos cognitivos de la terapia apuntan a la relevancia del significado (Beck, 1979), su formación, desarrollo, disfuncionalidad y posibilidades de cambio. En este enfoque lo más relevante no es la conducta, la emoción, la motivación o la mera cognición, sino el significado (Ruiz Sánchez et al, 2013).

La terapia cognitivo-conductual (TCC) ha mostrado ser el desarrollo teórico más sólido disponible hasta la fecha, y es la psicoterapia de referencia en el enfoque de la psicología contemporánea (Echeburúa et al, 2010). En el método terapéutico cognitivo se desarrolla un trabajo recorriendo un camino que va desde la conceptualización hasta la intervención, en el caso concreto. La conceptualización supone iniciar su proceso diagnóstico.

El modelo de atención de la Clínica de Psicología Utec, a través de los años, ha presentado una connotación cada vez más importante por el crecimiento estudiantil de la carrera de Licenciatura en Psicología, motivo por el cual ha sido necesario sistematizar un proceso de atención dirigido a la comunidad educativa en particular y a la sociedad salvadoreña en general. Tales procesos son tanto de forma como de fondo en los campos metodológico, administrativo y disciplinario, de acuerdo con entrevista realizada a la que fungió como encargada de la Clínica hasta diciembre del 2014, Licda. Claudia Mercedes Membreño.

Surge la importancia de documentar y concretar los procesos de formación académica y práctica clínica con pacientes reales. La naturaleza y el espíritu de la clínica se basa en la atención a la población que más lo necesita y que se acerca a solicitar el apoyo; y también en que funcione como un centro de prácticas, donde nuestros estudiantes de los últimos años de la carrera en Psicología realizan sus 
prácticas clínicas, convirtiéndose así en la actualidad en una clínica-escuela de atención psicológica.

La Clínica Psicológica cumple con dos criterios: la proyección social, por la atención a la población salvadoreña que lo solicita, y la formación de calidad de los estudiantes, futuros profesionales en el área. Es por eso que los esfuerzos en general empujan a inducir y formar disciplina en el manejo de la atención, proceso que se describe en dos áreas basadas en los criterios de administración y de enseñanza donde los estudiantes practicantes, denominados terapeutas, se rigen por la atención y logística de funcionamiento del centro de prácticas.

El proceso general, antes de iniciar el ciclo, se hace el análisis y preparación de materiales y recursos necesarios que se utilizarán en base a los siguientes puntos: número de alumnos en las asignaturas que tienen como requisito la práctica clínica; número de clínicas y de horas disponibles para brindar atención; número de solicitudes de los demandantes del servicio; recursos disponibles y los necesarios para iniciar el proceso; establecimiento de coordinaciones entre los entes rectores (director de escuela, encargada del centro de prácticas, docentes de las materias pertinentes y encargada de proyección social de la facultad) para coordinar el proceso de enseñanza y aprendizaje, así como el servicio a los solicitantes; establecimiento de fechas de inicio de las prácticas por los estudiantes; instrucción e indicaciones generales a los practicantes que se deben desarrollar en beneficio de su formación y para la atención al cliente.

El desarrollo de la Clínica de Psicología de la Utec ha sido un proceso de generar nuevas estrategias o fortalecimiento logístico de las existentes, para un mayor impacto, tanto en el aprendizaje de los estudiantes como en el apoyo de las situaciones de interés del usuario, por la tendencia del crecimiento de la carrera y la alta oferta (terapeutas) y demanda (usuarios solicitando atención) en la práctica y la atención.

De acuerdo con la descripción anterior, metodológicamente se percibe una alta tendencia en la práctica y el manejo terapéutico de la aplicación del modelo teórico cognitivo conductual, sustentado en el registro clínico (expedientes clínicos) y en los procesos de enseñanza para la atención de los usuarios en la resolución de sus necesidades y de las dificultades que ameritan atención psicológica, influenciado por la experiencia y conocimiento de los docentes a cargo de las materias que requieren el ejercicio práctico en ambiente y usuarios con dificultades reales. Por lo tanto, se establece como premisa, o se presume, que la inclinación de la atención de la clínica-escuela hasta la fecha se rige por el modelo cognitivo conductual en la enseñanza multimodal al estudiante y en la atención a los usuarios.

El modelo de atención psicológica tiene a su base el empleo de diversas etapas que se desarrollan en forma secuencial (ver figura 1). En cada una de ellas intervienen diversos actores en la ejecución del proceso; unos en la fase de planificación y organización, como lo es el encargado de la Clínica; otros con el apoyo administrativo en la recepción y atención de pacientes y estudiantes; y otros a través de su participación como terapeutas, brindando el servicio de atención psicológica. Estos últimos son formados y asesorados por un equipo de docentes responsables de la orientación académica. Asimismo, los estudiantes requieren en su proceso de atención del uso de diversos formularios (Anexo 8): Contrato terapéutico para niños y adultos, Expediente clínico, Notas administrativas, Notas evolutivas y Notas de asesorías. 
RECEPCIÓN EVALUACIÓN DIAGNÓSTICA EXPLORACIÓN DIAGNÓSTICO PSICOTERAPÉUTICA INTERVENCIÓN ORIENTACIÓN TERAPÉUTICA PEDAGÓGICA

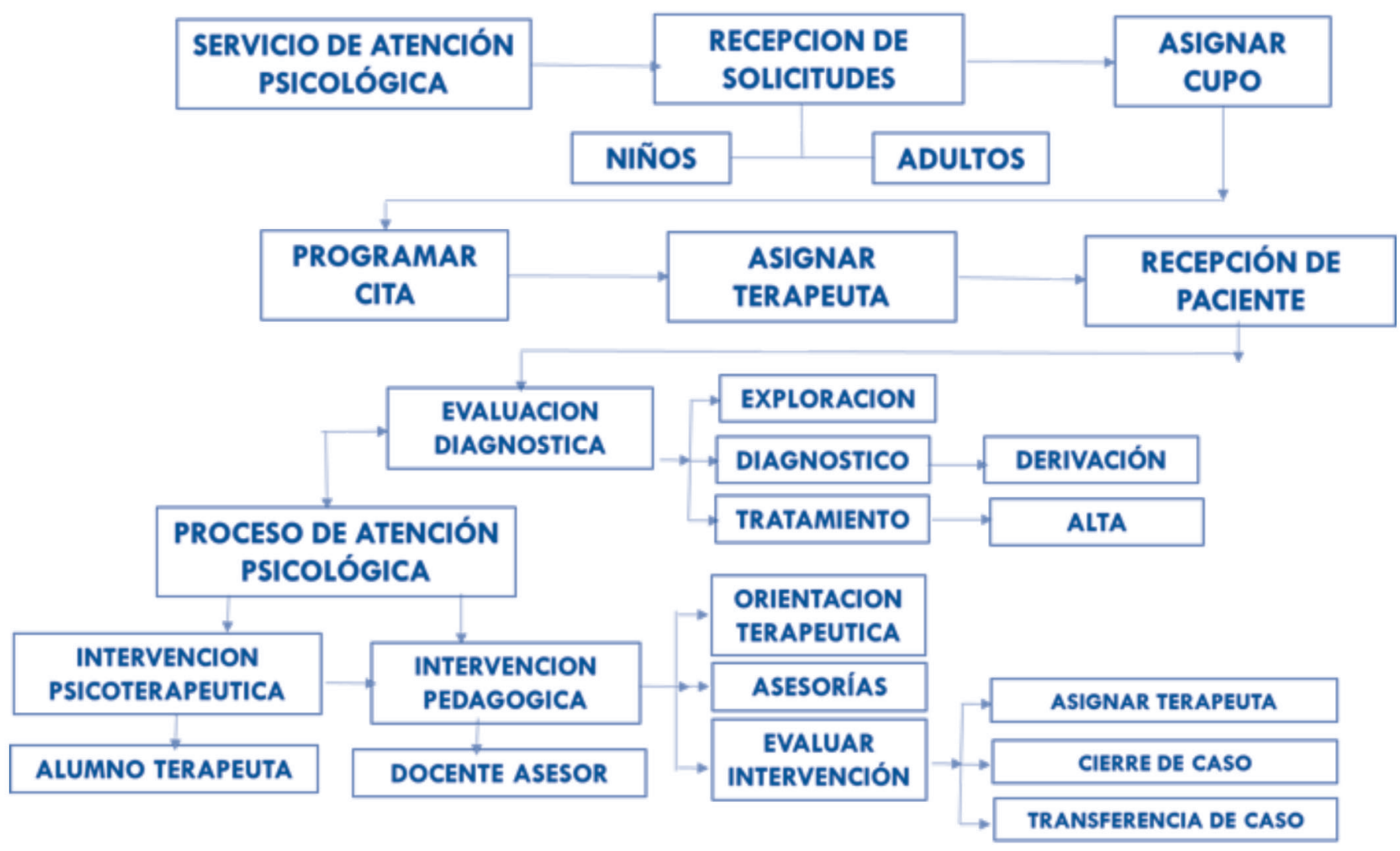

Figura 1.

Diagrama de flujo del proceso de atención de la Clínica Psicológica. Elaboración propia a partir de las experiencias de la Clínica Psicológica por el equipo de investigación.

En los últimos cinco años (del 2009 al 2013), la clínica de psicología de la Utec ha contado con estadísticas sistematizadas sobre la actividad del servicio de atención psicológica. En este período se han atendido a 1.310 usuarios. En la tabla 3 se resumen las características demográficas de los usuarios con referencia a las variables de sexo, edad y número de consultas por paciente. 
Tabla 3.

Características demográficas de los usuarios de la Clínica Psicológica Utec 2009-2013

\begin{tabular}{|c|c|c|c|c|c|}
\hline $\begin{array}{c}\text { Características } \\
\%\end{array}$ & $\begin{array}{c}2009 \\
n=211\end{array}$ & $\begin{array}{c}2010 \\
n=252\end{array}$ & $\begin{array}{c}2011 \\
n=295\end{array}$ & $\begin{array}{c}2012 \\
n=261\end{array}$ & $\begin{array}{c}2013 \\
n=291\end{array}$ \\
\hline Masculino $n$ & $90(42,7)$ & $124(49,2)$ & $141(47,8)$ & $126(48,3)$ & $143(49,1)$ \\
\hline Femenino $\mathrm{n}$ & $121(57,3)$ & $128(50,8)$ & $154(52,2)$ & $135(51,7)$ & $148(50,9)$ \\
\hline Media de edad & 19.9 & 19.2 & 20.4 & 19.4 & 18.5 \\
\hline Media de consultas & 6.5 & 4.9 & 5.8 & 7.8 & 7.6 \\
\hline
\end{tabular}

Fuente: Elaboración propia a partir de las experiencias de la clínica psicológica por el equipo de investigación.

De acuerdo con lo que se ilustra en la tabla anterior, la mayoría corresponde al sexo femenino $(52,4 \%)$ y el resto al masculino (47,6 \%). La media de edad de los usuarios de la clínica de psicología de la Utec es de 19.5 años, y el número promedio de consultas por paciente fue de 6.6.

El diseño de estudio de las buenas prácticas de la clínica de psicología de la Utec fue un enfoque multimodal, porque recolecta, analiza y vincula datos cuantitativos y cualitativos en un mismo estudio con una serie de investigaciones para responder a un planteamiento del problema. La combinación entre los enfoques cuantitativos y cualitativos se puede dar en varios niveles. La mezcla puede ir desde cualificar datos cuantitativos, o cuantificar datos cualitativos, hasta combinar ambos enfoques en un mismo estudio. Así, se puede desde generar un instrumento cuantitativo fundamentado en datos cualitativos hasta combinarse categorías de información de recolección cualitativa con datos continuos, en un análisis estadístico. Los diseños cuantitativos-cualitativos pueden ayudar a poner en práctica lecciones aprendidas en ambos enfoques, mantiene cerca del fenómeno estudiado y predice un sentido de entendimiento más completo (Hernández, Fernández y Baptista, 2006).

\section{Método}

Los participantes en la investigación fueron los siguientes:

- Estudiantes inscritos, a nivel de pregrado de la carrera de Licenciatura en Psicología de la Utec en el 2014, en las asignaturas de Tratamiento Psicológico Infantil ( $n=47$ ), Tratamiento Psicológico del Adulto $(n=138)$, Prácticas Psicológicas I ( $n=51$ ) y Prácticas Psicológicas II $(n=77)$, correspondiente al cuarto y quinto año de la carrera.

- Estudiantes inscritos en proceso de graduación. Del proceso 01-2014 $(n=43)$ y del ciclo 02-2014 $(n=63)$.

- Estudiantes de último ciclo de la carrera, en la asignatura Psicología Organizacional II ( $n=73)$.

- Docentes de la Escuela de Psicología de la Utec $(n=12)$; diez psicólogos de la cátedra de especialidad clínica y quince de otras unidades de la universidad que poseen formación profesional en el área clínica.

- Entrevista a usuarios o pacientes de la Clínica Psicológica $(n=42)$, que evaluaron el servicio psicológico, durante los ciclos 01 y 02 de 2014. 
Tabla 4.

Muestra de sujetos participantes

\begin{tabular}{ccc}
\hline Muestra & Frecuencia (F) & Porcentaje \\
\hline Alumnos pregrado & 386 & 70 \\
Alumnos egresados & 106 & 20 \\
Pacientes & 42 & 7 \\
Profesionales Psicología Clínica & 12 & 3 \\
Total & $\mathbf{5 4 6}$ & $\mathbf{1 0 0}$ \\
\hline
\end{tabular}

Fuente: Elaboración propia a partir de las experiencias de la clínica psicológica por el equipo de investigación.

\section{Instrumentos}

Seutilizaronseis instrumentos de recolección de información. Se realizaron entrevistas a docentes, estudiantes y usuarios de la Clínica de Psicología. Los instrumentos utilizados fueron los siguientes.

- Cuestionario de satisfacción de estudiantes de $4 .^{\circ}$ y $5 .^{\circ}$ años de la carrera de Psicología. Asignaturas de Especialidad Clínica. Este instrumento contiene ocho preguntas de respuesta cerrada en modalidad de escala Likert, con cuatro opciones de respuesta que van desde excelente, buena, regular hasta mala. Se incluyen en la pregunta nueve espacios para expresar sugerencias con relación a cómo mejorar el servicio de la Clínica Psicológica. Finalmente, hay un espacio para que escriba sus comentarios u observaciones generales sobre la clínica.

- Cuestionario de satisfacción y expectativas de estudiantes egresados en proceso de graduación. Este instrumento contiene cuatro apartados para evaluar áreas o temas de interés, competencias que desea fortalecer, evaluación de los servicios de la clínica y de los proyectos externos. Contiene 47 preguntas cerradas de elección libre, dando opción de seleccionar una o más respuestas. Cada apartado incluye un espacio para que los estudiantes egresados escriban sus comentarios o sugerencias.

- Técnica Foda, aplicada a estudiantes de la asignatura de Psicología Organizacional II, para evaluar el servicio de atención de la Clínica Psicológica. Este instrumento se administró en forma colectiva al grupo de estudiantes dentro del salón de clases. Se formaron equipos de trabajo, se les dio las indicaciones y proporcionó una hoja de papel para que elaboraran su diagnóstico Foda.

- Entrevista a docentes de la Escuela de Psicología sobre los servicios de la Clínica Psicológica. Este instrumento contiene seis preguntas abiertas, en las cuales los docentes del área de Psicología Clínica describen su experiencia laboral en el ejercicio de la profesión. Al final del instrumento se incluye un espacio para escribir comentarios y evaluar los servicios y la orientación formativa de los alumnos.

- Cuestionario a usuarios o pacientes de la Clínica Psicológica. Se aplicaron cuestionarios de satisfacción a los usuarios de la clínica en los ciclos 01-2014 y 02-2014. Este instrumento contiene 24 preguntas. Se utilizó el tipo de escala de Likert. Cada pregunta con tres opciones de respuesta, siendo estas: excelente, buena y regular. Adicional a cada respuesta hay un apartado para escribir comentarios y observaciones. Al final también se incluye un espacio para escribir comentarios o recomendaciones abiertas, por parte de los usuarios, para mejorar el servicio de la clínica.

- Base de datos de pacientes atendidos en la clínica psicológica en el período correspondiente del 2009 al 2013. La información recolectada de los expedientes clínicos de los pacientes se tiene registrada en una base de datos. Las variables que se han incluido en el presente estudio a lo largo de este período son datos generales como edad, sexo, motivo y número de consultas. 


\section{Resultados}

Se presenta un análisis descriptivo de la evaluación del nivel de satisfacción con el servicio que brinda la Clínica de Psicología de la Utec. Se incluyen las frecuencias y porcentajes de los niveles de satisfacción de los estudiantes/ terapeutas, usuarios/pacientes, docentes y estudiantes de Ios últimos años de la carrera y egresados de esta. Asimismo, se incluye el resultado de la evaluación que realizaron los catedráticos de la especialidad clínica, quienes tienen a su cargo la formación de nuestros estudiantes.

\section{Resumen de análisis de resultados de Cuestionario de satisfacción de estudiantes de $4 .^{\circ}$ y $5 .^{\circ}$ años de la carrera de Psicología. Asignaturas de Especialidad Clínica}

De acuerdo con la opinión de los estudiantes de la carrera, en relación con la calidad que brinda la Clínica de Psicología, se destacan los siguientes datos: $59 \%$ expresa que la calidad del servicio que brinda la clínica de Psicología de la Utec se encuentra entre bueno y excelente. $69 \%$ considera sentirse satisfecho de manera general, o total, con la formación en el área clínica ofrecida por la Utec. El $45 \%$ de los estudiantes de la carrera de Psicología se muestra satisfecho y de acuerdo con sus necesidades personales. El $65 \%$ de los estudiantes recomendaría el servicio de la Clínica de Psicología a un amigo. El $86 \%$ de los estudiantes se muestra satisfecho con las asesorías recibidas por parte de sus docentes. El $41 \%$ manifiestan que los conocimientos adquiridos en la carrera le han ayudado para atender eficazmente a sus pacientes. El 77 \% se muestra satisfecho con la formación en el área clínica ofrecida en la carrera. El 72 \% opina que utilizaría los servicios de la clínica de Psicología si necesitara dicho servicio.

Resumen de análisis de resultados de Encuesta de satisfacción y expectativas de estudiantes egresados en proceso de graduación

De acuerdo con la opinión de los egresados de la carrera de Psicología, en relación con la satisfacción que brinda la clínica, se destacan los siguientes datos: $36 \%$ prefiere la psicología clínica como un área de especialidad, seguido por las opciones de psicología laboral y organizacional (19 $\%) ; 15 \%$ considera que las competencias que se deben fortalecer son las relacionadas con las técnicas de violencia intrafamiliar y de género, seguido por las competencias del área evaluación psicométrica en recursos humanos y en el área forense de elaboración de peritajes (11\%); 38 $\%$ opina que deben ser tratados con amabilidad y respecto -entre regular y buena- por el personal administrativo de la escuela. El 39 \% manifiesta satisfacción —entre buena y excelente- con los horarios asignados para su práctica clínica. El 41 \% expresa que la asesoría brindada por el docente de su asignatura ha sido entre buena y excelente. El $44 \%$ de los encuestados considera que las condiciones de limpieza y orden de las clínicas de psicología se encuentran entre buenas y excelentes. El $45 \%$ manifiesta beneficio y utilidad en la realización de prácticas en la Clínica de Psicología —entre bueno y excelente-. El 40 \% comentó que se encuentra satisfecho referente a la atención y trato al estudiante por parte de la administración de la clínica.

\section{Análisis de resultados de la técnica Foda, aplicada a es- tudiantes de la carrera de Psicología, para evaluar el servicio de la Clínica Psicológica Utec}

\section{Fortalezas}

Capacidad para atender y conocer diversos problemas de Ios pacientes. Permite realizar prácticas de estudiantes. El contacto es directo con el paciente. Buen ambiente para practicar. Apoyo a personas que lo solicitan y ayuda a los demás con sus problemas. Se brinda el servicio de forma gratuita. Se da atención profesional. Hay credibilidad por parte del paciente. Permite el desarrollo. Aprendizaje y crecimiento académico de los estudiantes. Se practican las técnicas de la entrevista. Se ponen en práctica las técnicas de evaluación y tratamiento. Permite conocer y practicar las terapias. Buena asesoría por parte del docente. Se fomentan valores, entre ellos el de la puntualidad y el secreto profesional. Posee material de apoyo, papelería necesaria y mobiliario.

\section{Debilidades}

Saturación de clínicas y tiempos prolongados para atender a los pacientes. Dificultad de horario. Consulta cada 15 días. Poca cartera de pacientes. No se hace propaganda acerca de la clínica, por eso no llegan pacientes. Falta de recursos 
lúdicos y didácticos. Falta de coordinación con el paciente y el terapeuta para notificar permisos o ausencias.

\section{Oportunidades}

Desarrollo de nuevos conocimientos y aprendizaje permanente. Promover la experiencia en el área clínica. Fomentar el crecimiento personal y académico. Poner en práctica la teoría y las terapias psicológicas. Ampliar los sectores de personas beneficiadas y ayudar a los más necesitados. Ampliar los servicios de consulta psicológica gratis. Mayor cobertura de atención. Mejorar la salud mental de la población. Promover mayor credibilidad de parte de los estudiantes. Fomentar responsabilidad por parte del estudiante. Fomentar la ética profesional.

\section{Amenazas}

Infraestructura no adecuada para el crecimiento de la carrera. Los grupos de práctica son muy grandes. Insuficientes las instalaciones físicas. Disminución de pacientes por la mala administración. Posibles repercusiones legales al terapeuta por mala atención. Falta de motivación por parte del paciente. Poca divulgación de la clínica.

\section{Análisis de la entrevista a docentes de la Escuela de Psicología sobre los servicios de la Clínica Psicológica de la Utec}

Al consultar a los profesionales de la Psicología sobre cuál es el modelo teórico que se enseña o utiliza en la formación de los estudiantes en las asignaturas del área clínica, opinaron lo siguiente: Modelo a través de prácticas clínicas, evaluaciones, diagnósticos y tratamientos clínicos. No lo tengo claro, ya que muchos dicen ser psicoanalistas, pero la realidad no lo son. No hay un modelo definido; creo que depende del enfoque que siga el docente, aunque traen un enfoque "médico" de algunas materias como Psicopatología. Enfoque genérico. Muy amplios los diversos enfoques. Ninguna en específico. Modelo cognitivo-conductual. Método socrático. Enfoque ecléctico. Esta perspectiva permite al estudiante incorporarlo en su práctica profesional, ya que los problemas de consulta clínica no siempre pueden ser abordados con un solo modelo. Aunque se propone como alternativa el enfoque ecléctico, se sugiere adoptar el Modelo Integrador. Ecléctico. Programación neurolingüística (PNL), cognitivo conductual, psicoanálisis. No hay un modelo claro y directo; depende del docente a cargo. La formación teórica está basada en el aprendizaje cognitivo conductual; el modelo no está claramente definido, pero está basado en competencias que se van dosificando, y estas se van poniendo en práctica. Práctica-Teoría-Práctica.

Los profesionales de la Psicología opinaron que las técnicas o herramientas que necesitan conocer o aprender los estudiantes en su formación clínica son las siguientes: entrevistas clínicas, estrategias de intervención, evaluación mental, elaboración de plan de tratamiento; manejo de test, manejo de entrevista clínica, elaboración de planes terapéuticos; redacción de informes psicológicos, presentación de resultados y encuadre de pruebas psicológicas.

Las competencias que deben de desarrollar son las siguientes: evaluar, diagnosticar a través de pruebas psicológicas y entrevista clínica para ofrecer tratamiento de acuerdo con el caso, implantar procesos de atención psicológica en salud mental a grupos; aplicación e interpretación de pruebas psicológicas, técnicas de entrevistas, manejo del DSM, elaboración de historias clínicas; condicionamiento clásico: técnicas de relajación profunda con imaginación inducida, desensibilización sistemática); condicionamiento operante: economía de fichas, algunas técnicas del condicionamiento encubierto, entrenamiento en habilidades sociales (EHS), entrenamiento de padres; técnicas cognitivas y de autocontrol: terapia racional-emotiva, entrenamiento en solución de problemas, la inoculación de estrés, métodos de autocontrol, entrenamiento en autoinstrucciones; técnicas de relajación, técnicas de autocontrol emocional, técnicas de modificación de la conducta, técnicas cognitivo-conductuales; entrevista, ética profesional, técnicas terapéuticas; ludoterapia, tamizajes de violencia, pruebas psicológicas, tipos y formas de diagnósticos; entrevistas de exploración, diagnósticos certeros, manejo de crisis; técnicas de intervención psicológica, manejo de oralidad y liderazgo grupal; tratamientos psicológicos a casos específicos; las técnicas cognitivas: aprendizaje de resolución de problemas; desarrollo de valores o dilemas morales, habilidades sociales, control del comportamiento agresivo, manejo de la ansiedad; técnicas conductistas: programas de refuerzo intermitente, intervalo fijo de variables, programas de ra- 
zón; técnicas de entrevista-investigación; teoría y práctica de aplicación y evolución de los instrumentos psicológicos; redacción de informes clínicos (análisis-síntesis).

Según opinión de los profesionales de la Psicología, los contenidos o conocimientos teóricos que deben poseer los estudiantes en el área clínica son estos: enfoques y corrientes psicológicas y aplicaciones psicoterapéuticas (Gestalt, conductismo, psicoanálisis, humanismo, bioenergética, conductual, PNL); enfoque cognitivo conductual, psicoterapia de enfoque breve, planes de intervención terapéutica, intervención en crisis; modelos psicopatológicos, psicoterapéuticos, diagnósticos, evaluación; diferentes formas de entrevistas (personales, por teléfono, email), abordamiento en situaciones de crisis, tratamiento a corto plazo, dificultades transversales; teorías de la personalidad, elaborar diagnósticos, diseñar tratamientos, desarrollar procesos terapéuticos, la entrevista; sensibilidad para conectarse empáticamente con la persona que busca ayuda; competencias de la entrevista clínica y diagnóstica; manejo del diálogo socrático; dominio de la o las técnicas seleccionadas; llevar a cabo el seguimiento de los casos; ética profesional, legislación relacionada con la práctica clínica psicológica, diseño, planificación, ejecución, y evaluación de programas de prevención primaria, secundaria y terciaria en salud mental; habilidades básicas de un terapeuta competente; evaluación de la eficacia del tratamiento psicoterapéutico; bibliografía, investigación clínica, farmacología, entrevistas clínicas, ética profesional; intervención en crisis (técnicas), abusos sexuales, violencia intrafamiliar, diversidad sexual, psicogeriatría; manejo de crisis, psicología hospitalaria y salud, psicología jurídica, manejo de duelos, terapia de parejas, manejo de suicidio y depresión; conocimiento sobre el origen y desarrollo del psiquismo humano; conocimiento sobre los criterios diagnósticos y cómo diagnosticar; conocimiento sobre técnicas de entrevista para abordar los casos; técnicas de comunicación no verbal, la entrevista clínica (adultos, niños, colaterales); estadística aplicada a la psicología (medidas de tendencia central), ortografía y redacción general, redacción de informes clínicos, técnicas de recolección de datos.

Según la opinión de los profesionales de Psicología, las competencias o habilidades que deben tener los estudiantes en el área clínica son lenguaje técnico, conocimiento de farmacología, redacción y lenguaje corporal; escucha activa, evaluaciones diagnósticas a través de pruebas psicológicas, elaborar informes psicológicos clínicos; creación de planes de tratamiento, conocimiento de técnicas de entrevista, saber diagnosticar, manejo de técnicas terapéuticas y enfoques psicológicos; habilidad para entrevistas clínicas, resolución de casos, creación de planes terapéuticos, diagnóstico de diferentes pruebas psicológicas; evaluar pacientes, elaborar diagnósticos, diseñar tratamiento, procesos terapéuticos, la entrevista (descuidan el análisis del contexto social y sus repercusiones en el origen y mantenimiento del problema; cuando el contexto no es valorado e incorporado como elemento que se debe modificar por la intervención psicoterapéutica, las técnicas por sí mismas no son suficientes para el éxito de la terapia); empatía en el terapeuta; habilidades básicas de escucha, reconocimiento de la respuesta verbal y las intenciones del terapeuta y del paciente; habilidades para establecer alianzas terapéuticas; conocimientos, técnicas y estrategias para recolectar, sistematizar y utilizar con un objetivo clínico psicoterapéutico la información el paciente, manejo de expediente clínico psicológico; responsabilidad, ética, compromiso con la carrera, leer; empatía, habilidad para desarrollar observación capacidad crítica y autocrítica, habilidad para hacer diagnósticos; hacer buenos diagnósticos; elaboración de informes y planes de hipótesis coherentes; terapia grupal; inteligencia emocional; habilidades cognitivas, razonamiento, análisis hipotéticos, abstracción; hacer buenos diagnósticos, elaboración de informes y planes de hipótesis coherentes, terapia grupal, inteligencia emocional, habilidades cognitivas (razonamiento, análisis hipotéticos abstracción; aplicar técnicas de evaluación para diagnósticos; elaborar informes psicológicos; elaborar planes de intervención individual y grupal; aplicar técnicas de contención emocional y manejo de crisis en casos de desastres naturales; tener espíritu investigador y proactivo; habilidades para comunicarse efectivamente, entrevistar, observar, analizar-sintetizar, redactar informes clínicos, aplicar terapias.

Algunas experiencias en su desempeño como docente o profesional en la atención clínica en la formación académica de los estudiantes de la carrera son elaboración de plan psicoterapéutico; hacer círculos de estudio, asesorías individuales a pequeños grupos, modelaje de los docentes; apoyo psicológico, redes sociales; apoyo en atención 
de víctimas de abuso sexual (comentario escuchado a los alumnos de la preespecialidad: "No se nos prepara para la atención de víctimas de abuso sexual"); presentación, ética, respeto e identificación con la Utec; planificación, trabajo en equipo; abuso sexual histórico de primer grado; la falta de interés de algunos estudiantes ante problemas reales de pacientes delicados, como suicidio, VIH.

De acuerdo a la experiencia de los docentes, se dosifican las competencias en pequeños pasos: alianzas terapéuticas, contrato, entrevista clínica inicial, historia clínica, aplicación de pruebas, examen mental, diseño de plan terapéutico, informe psicológico. Varios estudiantes han seguido su formación en el área clínica y están especializándose con maestrías Es necesario que haya docentes que orienten y acompañen al estudiante durante la realización de sus prácticas en la Clínica psicológica, esto de manera profesional, y que no solo se dediquen a regañarlos por sus malos procedimientos.

En resumen, los profesionales de la psicología han expresado sobre la Clínica de Psicología de la Utec: está bien organizada, tiene áreas ventiladas, iluminadas, ordenadas y recursos lúdicos; la atención es gratuita, tiene mucha demanda, se hace labor social; apoyo al aprendizaje, ayuda a la comunidad, buen trabajo terapéutico, comodidad; condiciones adecuadas, credibilidad del paciente; demanda, credibilidad, espacio; sistematización de procesos, orden; las actitudes de los estudiantes se fortalecen y se practican Ios valores: integridad, liderazgo, responsabilidad; el soporte técnico de los docentes como tutores; la infraestructura para que el estudiante se empodere y practique con monitoreo cercano; hay clínica; tiene cierto nivel de organización; hay demanda de estudiantes en prácticas y pacientes.

No obstante, los profesionales de la psicología han expresado que en la Clínica de Psicología se brinda poca asesoría a los estudiantes, el espacio es reducido, los docentes no apoyan al terapeuta; muchos terapeutas y pocas clínicas; extender horarios de consulta; mala administración, pocas clínicas, poco personal; poco espacio, mucha demanda y muy largo el tiempo para la atención cada quince días; ocho sesiones evaluando el caso antes de comenzar a "tratarlo"; según la metodología que se va a emplear, el número de sesiones disponibles para evaluar el caso no debe de pasar de tres; para evaluación clínica en Psicología, con fines diagnósticos, no se cuenta con una amplia batería de test psicológicos, que se puedan cubrir en tres a cuatro sesiones, por ejemplo, si se va a aplicar un WISC-R; existe un compromiso débil, hay falta de identificación terapéutica, poca planificación; organización, exclusividad de casos, no se atiende a todos los casos; variabilidad de direcciones en coordinación; trabajan independiente con el docente titular, por lo que hay tres personas diferentes encargadas de la nota del estudiante; no buscan convenios con nuevas instituciones; los estudiantes realizan actividades en los centros de prácticas que no son acordes con la actividad terapéutica; son inflexibles en el manejo del expediente; mucha población estudiantil y pocas clínicas; algunos problemas en la administración; utilización de una misma secretaria para la clínica y la escuela; espacio reducido, pocas clínicas y horarios cada quince días; destinar una persona que específicamente lleve el control de expedientes; las personas abandonan los tratamientos; pocas clínicas para la demanda estudiantil; no cuenta con gabinete de pruebas psicológicas.

Algunas recomendaciones que han brindado los profesionales de la psicología son las siguientes: se tiene que mejorar los espacios físicos para la atención psicológica, mejorar el trato a los estudiantes; demasiado trabajo para la administración, muchos terapeutas; la administración de la clínica debe tener conocimientos amplios de clínica y tener solvencia moral y profesional, ser mediadora; los estudiantes tienen oportunidad de realizar buenas prácticas clínicas; es importante una reunión general con los docentes para tratar asuntos de la clínica y que los grupos de alumnos de la especialidad sean de 35, para que el aprendizaje sea mejor aprovechado por el alumno; los estudiantes deben hacer prácticas fuera de clínica; los primeros niveles deben estar dentro de la clínica, y que los alumnos de $4 .^{\circ}$ y $5 .^{\circ}$ años realicen prácticas fuera; es necesario respetar a los estudiantes, no confundir la disciplina con autoritarismo; el respeto se forma con el buen trato, inteligencia emocional y habilidad social. A los profesionales les parece muy adecuada la normativa para los estudiantes en sus prácticas.

\section{Resultados}

Según la opinión a los usuarios o pacientes, en relación con los servicios que brinda la Clínica de Psicología, se destacan 
los siguientes datos: $76 \%$ de los pacientes evalúan excelente el tiempo de espera en respuesta a su solicitud de servicio; $91 \%$ de los usuarios considera que en la recepción fueron atendidos en forma amable y respetuosa; $83 \%$ de los terapeutas les informó acerca de las normas y reglas de la clínica de psicología; 86 \% considera excelente la ubicación de la clínica psicológica; $100 \%$ de los usuarios considera que el terapeuta lo atendió en forma amable; 60 \% considera excelente que el trato recibido vía telefónica fue con amabilidad; 76 \% está satisfechos con su horario de consulta de atención psicológica; 62 \% evalúa excelente su tiempo de espera desde que presentó su solicitud de atención psicológica; 86 \% evalúa excelente el nivel de confianza y la ayuda brindada por el terapeuta; $71 \%$ se encuentra satisfecho con el tiempo de espera en la sala de consulta, para la atención psicológica; 76 \% evalúa excelentes las actividades (técnicas) utilizadas por el terapeuta; $57 \%$ considera excelentes las orientaciones psicológicas que le dio el terapeuta, y $43 \%$ evalúa como buenas las orientaciones; $55 \%$ de los usuarios considera excelente el número de sesiones de atención por parte del terapeuta a su caso, y $45 \%$ lo considera bueno; $79 \%$ considera excelente el orden y la limpieza de las instalaciones en la Clínica de Psicología; 81 \% evalúa excelente el beneficio y la utilidad del servicio de atención psicológica recibida; $76 \%$ de los usuarios recomendaría el servicio de la clínica psicológica a sus familiares, amigos o compañeros; $81 \%$ califica excelente la calidad del servicio psicológico recibido; $71 \%$ de los usuarios considera que, en general o totalmente, sí encontró la clase de servicio que buscaba. $85 \%$ de los usuarios considera que la clínica ha satisfecho sus necesidades en forma total o general; $90 \%$ de los usuarios probablemente recomendaría la clínica a un amigo si necesitará una ayuda psicológica similar; $71 \%$ de los usuarios considera estar muy satisfecho con la ayuda psicológica ofrecida; $71 \%$ de los usuarios considera que el servicio le ha ayudado mucho a resolver sus problemas, y $29 \%$ considera que le ha ayudado bastante; $95 \%$ de los usuarios está satisfecho con el servicio psicológico recibido; 95 \% de los usuarios considera que sí, o probablemente sí, volverían a la clínica en busca de ayuda.

En relación con el tiempo de asistir como paciente a la clínica psicológica, los usuarios manifestaron lo siguiente: $36 \%$ estar menos de un mes, $19 \%$ de uno a tres meses y $45 \%$ más de tres meses.
Al preguntar a los usuarios cómo se habían enterado de los servicios que brinda la Clínica de Psicología, 60 \% manifestó que fue a través de amistades, $36 \%$ a nivel personal y $5 \%$ por referencia de centros educativos.

\section{Análisis de resultados de encuesta a usuarios 0 pacientes de la Clínica Psicológica}

En la revisión de la base de datos de los expedientes clínicos de los pacientes atendidos en la Clínica de Psicología, en el período del 2009 al 2013, se destacan los siguientes resultados: el principal motivo de consulta de los pacientes a la clínica es por problemas de conducta; agresividad y aprendizaje (32,8\%); le sigue problemas personales, proceso profesional y baja autoestima (18,3\%); a continuación están los problemas familiares y de pareja $(12,3 \%$ y depresión y ansiedad (12,1\%).

En la revisión de la base de datos del registro de los expedientes clínicos de los pacientes atendidos en la Clínica de Psicología, se observa el número de consultas brindadas a los pacientes, como sigue: $40,2 \%$ recibieron de una a cuatro consultas psicológicas. $24,6 \%$ recibieron de cinco a ocho consultas. 22,0 \% recibieron de nueve a doce consultas.

\section{Discusión}

El fenómeno de la salud mental es de suma importancia en la situación de crisis delincuencial que vive el país posterior a la firma de Acuerdos de Paz (1992). Diversas instituciones han aunado esfuerzos en pro de la salud mental. Particularmente en la Utec, desde 1984, se ha brindado el servicio de atención psicológica a la comunidad universitaria y al público, poniendo al servicio de la sociedad salvadoreña la Clínica Psicológica en coordinación con el Ministerio de Salud.

En forma paralela, la carrera de Psicología a escala nacional se ha fortalecido gracias al respaldo de la legislación en el área de salud a través de organismos gubernamentales que dictan las políticas de esta. Entre ellas se mencionan la Política Nacional de Salud Mental del Ministerio de Salud, en el 2011; la creación de la Junta de Vigilancia de la Profesión en Psicología (JVPP), en febrero 1987; y las disposiciones 
emanadas del Código de Salud en el 2001, con relación a que la JVPP goza de autonomía en su función y sus resoluciones, pero está sujeta a disposiciones del Código de Salud, dentro del Consejo Superior de Salud Pública (Código de Salud, Arts. 5, 9 y 10, 2001).

La presente discusión integra los siguientes elementos: evolución histórica, crecimiento de la carrera de Psicología, necesidad permanente de atender la problemática de la salud mental y el respaldo de la normativa legal que sustenta los servicios de atención psicológica en el país.

No podemos dejar de mencionar que el fundamento teórico sobre el cual se realizó el presente estudio es desde la perspectiva de la Psicología bajo un modelo ecléctico con implicaciones y aplicaciones de diferentes disciplinas 0 modelos teóricos, tradicionales o actuales. La salud mental es tipificada de diversas formas, y se considera que es más que la simple ausencia de trastornos mentales, afectaciones o discapacidades globales. Según el Comité de Salud Mental de la Organización Mundial de la Salud (OMS, 2011), la salud mental se define como: "El goce del grado máximo de salud que se puede lograr, es uno de los derechos fundamentales e inalienables del ser humano, sin distinción de raza, religión, ideología política o condición económica y social".

Se presentan a continuación los resultados más relevantes del estudio de buenas prácticas de la Clínica de Psicología Utec. La mayoría de estudiantes de la especialidad clínica de la carrera de Psicología de la Utec considera que la calidad del servicio que brinda la Clínica Psicológica es entre buena y excelente. La mayoría de estudiantes recomendaría los servicios que brinda la Clínica de Psicología de la Utec a un amigo y utilizaría los servicios de la clínica de manera personal. La mayoría de estudiantes se muestra satisfecha con la asesoría y la formación recibidas en el área clínica por parte de los docentes.

Al evaluar la satisfacción y expectativa de los estudiantes egresados, en proceso de graduación, sobre la calidad de servicio de la clínica psicológica, observamos que la mayoría se mostró satisfecha con el horario asignado a su práctica clínica y la asesoría brindada por los docentes de la especialidad. Asimismo, la mayoría considera de beneficio y utilidad la realización de prácticas en el área clínica.
La información anterior es el resultado del sondeo realizado a estudiantes de la carrera de Psicología a nivel de pregrado y a egresados para conocer su nivel de satisfacción y logro de expectativas por el servicio de la clínica psicológica. Es decir, estos resultados son parte de los hallazgos al entrevistar a los terapeutas psicológicos en formación. Asimismo, se analizan los resultados de la técnica Foda en el ámbito institucional. Los estudiantes enfocan como fortaleza en la clínica el hecho de que se les permite realizar sus prácticas en contacto directo con los pacientes. Asimismo, permite el desarrollo, aprendizaje y crecimiento académico de los estudiantes, con prácticas técnicas de entrevistas, la práctica de evaluación y tratamiento, y por conocer y practicar diferentes tipos de técnicas y terapias según la necesidad del paciente. Los estudiantes identifican que la clínica fomenta valores, permite proyectar a la Utec a través de un servicio gratuito a la socieda y que existe credibilidad por parte de los pacientes.

No obstante, la clínica se podría fortalecer promoviendo nuevos conocimientos y aprendizajes permanentes en elárea clínica a través de nuevas teorías y terapias psicológicas. De igual forma, se podría brindar mayor cobertura de atención mediante la divulgación de los servicios que brinda la clínica. En el futuro, las infraestructuras de las instalaciones físicas de la clínica resultarán insuficientes debido al crecimiento de la carrera y al aumento en la demanda de los pacientes que solicitan el servicio psicológico.

El modelo utilizado para la formación teórica de los terapeutas de la clínica psicológica Utec, por parte de los profesionales de especialidad en Psicología Clínica, está basado en el aprendizaje de competencias para los terapeutas psicológicos. También se practica el modelo cognitivo conductual con diferentes tratamientos y técnicas. Y en consenso con los entrevistados, el enfoque es ecléctico. Este modelo permite al estudiante incorporar en su práctica profesional el abordaje directo con pacientes que requieren el servicio de la Clínica.

Resultan interesantes las técnicas y herramientas que los profesionales de la psicología sugieren que los alumnos terapeutas en formación deben conocer, que son las siguientes: técnicas de entrevista, estrategias de intervención, evaluación mental, elaboración de plan de 
tratamiento terapéutico, redacción de informes psicológicos, manejo de test psicológicos, diagnosticar a través del Manuel Diagnóstico y Estadístico de Trastornos Mentales (DSM-5), aplicar tratamientos según el caso presentado, implantar procesos de atención en salud mental individual y grupal, técnicas de relajación, técnicas de condicionamiento, prácticas de terapias racional-emotivas, entrenamiento de habilidades sociales (EHS), entrenamiento de padres y madres, ludoterapia, tamizajes de violencia, desarrollo de valores o dilemas morales e investigación.

Los profesionales clínicos sugieren que los terapeutas de la Clínica deben poseer los siguientes conocimientos teóricos, básicos para su buen ejercicio profesional: diversos enfoques psicológicos y sus aplicaciones psicoterapéuticas, psicología hospitalaria, manejo de duelos, suicidios y depresión, desarrollo del comportamiento humano, estadística aplicada a la psicología, redacción de informes clínicos.

Los profesionales clínicos sugieren que las competencias que se deben desarrollar en los terapeutas en formación en la clínica terapéutica son los siguientes: lenguaje técnico psicológico, interpretación del lenguaje corporal, escucha activa y empática, evaluaciones diagnósticas, elaboración de informes psicológicos, creación de planes de tratamiento, entrevistas clínicas, resolución de casos, creación de planes estratégicos, análisis del contexto social y sus repercusiones en el origen y mantenimiento del problema, habilidad para establecer alianzas terapéuticas, manejo de expediente clínico psicológico, responsabilidad, ética, compromiso hacia la carrera, inteligencia emocional, habilidades cognitivas, razonamiento, análisis hipotético, abstracción, manejo de crisis en casos de desastres naturales, espíritu investigador y proactivo, comunicarse efectivamente.

La mayoría de usuarios o pacientes de la clínica psicológica de la Utec considera satisfactorio, o muy satisfactorio, el tiempo de espera para ser atendidos. La mayoría opinó que el terapeuta le brindó adecuada información sobre las reglas y normas de la clínica, y fue atendido en forma amable. También los pacientes se muestran satisfechos con relación a la hora que se les programó para recibir sus consultas, y consideran adecuado el tiempo de espera en la sala de la clínica previo al inicio de la consulta. Expresan gran satisfacción por la ayuda psicológica brindada por el terapeuta. Esto referido a la satisfacción con las actividades y orientaciones psicológicas que le brindó el terapeuta. La mayoría de los usuarios expresaron su satisfacción por el número de sesiones que le dedicó el terapeuta a su caso y, asimismo, opinan que recomendarían utilizar el servicio de la clínica de psicología de la Utec con algún amigo, conocido o familiar.

La mayoría de los pacientes califica los servicios de la clínica como excelentes. Asimismo, la mayor parte de los usuarios considera que encontró en la clínica la clase de servicio que buscaba. De igual forma, a satisfecho sus necesidades. Todo lo anterior es congruente, en el sentido de que los usuarios consideran que si un amigo necesitara de una ayuda similar recomendaría la Clínica Psicológica Utec. Se confirma la opinión de la mayoría de los usuarios con relación a que si está satisfecho con la ayuda recibida y los servicios le han ayudado en forma eficaz a hacer frente a sus problemas. Finalmente, se confirma que el o los pacientes, si tuvieran que buscar ayuda, volverían sin dudar a solicitar nuevamente los servicios de la Clínica Psicológica.

En relación con las terapias psicológicas efectivas ofrecidas por el terapeuta, se encuentran los siguientes resultados: la mayoría tuvo una atención psicológica de tres meses o menos, y para una minoría de pacientes su asistencia fue de más de tres hasta seis meses; solo se incluyen los casos que finalizaron su tratamiento. Este último dato es apoyado por Labrador, Estupiñá \& García Vera (2010), en relación con que en la CUP de la UCM, en lo referido a las variables terapéuticas, sobre el número de sesiones de evaluación, estas oscilan entre 0 y 14 sesiones, siendo la media y la moda 4 . El $83 \%$ tuvo cuatro o menos sesiones de evaluación y solo el $3 \%$ más de tres sesiones. Relacionado con el número de sesiones de tratamiento, solo se incluyen los casos que han finalizado el tratamiento, ya fueran alta, seguimiento o evaluación postratamiento. El número de sesiones osciló entre 0 y 66 , siendo la mediana 11 . El 75,3\% de los sujetos recibió 18 o menos sesiones de tratamiento.

Finalmente, se consultó a los usuarios sobre cómo se enteraron de los servicios que brinda la Clínica Psicológica de la Utec; la mayoría expresó que obtuvo información a través de amistades, y otro sector considerable expuso que hizo una visita personalmente. 
Los hallazgos en la revisión cronológica de los expedientes clínicos de los pacientes atendidos en la Clínica permiten conocer, a partir de los registros en la base de datos de 1.310 pacientes atenidos, entre los años 2009 a 2013, los siguientes puntos relevantes. La mayoría de los motivos de consulta por los que asisten los pacientes son problemas de conducta, de aprendizaje y de agresividad. Otro factor importante son los problemas personales, la búsqueda de orientación profesional y los problemas de baja autoestima. Le siguen los problemas familiares y de pareja, y muy cercano los problemas de ansiedad y depresión. Labrador, Estupiñá y García Vera (2010) señalan que la distribución de diagnósticos de los pacientes, considerado según los criterios del DSM IV y DSM IV TR (APA, 1995, 2000), se destacan como los de mayor relevancia los trastornos de ansiedad (31,89\%), sin diagnóstico (14,84\%), trastorno del estado de ánimo (9,46 \%) y problemas de relación (9,81\%), seguido por una serie de diagnósticos en menor proporción, que componen alrededor del $45 \%$ restante. Sobre este mismo fenómeno, Martorell y Carrasco Ortiz encontraron que en el tipo de demandas atendidas, tomando como base el DSM IV-TR (APA, 2010), se observan los diagnósticos realizados en el Servicio de Psicología Aplicada (SAP) de la Facultad de Psicología de la Uned. Mayoritariamente, las principales demandas son debidas a los trastornos del estado de ánimo (10,9\%) y los trastornos de ansiedad (31,3\%). Se destacan otros problemas de atención clínica, entre los que cabe mencionar los problemas de relación paterno-filiales y conyugales $(24,7 \%)$.

La mayoría de los pacientes atendidos en la Clínica Psicológica de la Utec se encuentra en el rango de los 5 a los 12 años, seguido por un grupo representativo de pacientes de los 20 a los 30 años, y en un tercer gran grupo se encuentran los de entre 13 y 19 años de edad. La mayor parte de los pacientes son mujeres $(51,8 \%)$. Este es el mismo hallazgo de Labrador, Estupiñá y García Vera (2010) y de Martorell y Carrasco Ortiz (2009).

A lo largo de los últimos cinco años (2009-2013), de los que contamos con estadísticas sistematizadas sobre la actividad del servicio de la Clínica de Psicología de la Utec, se han atendido a 1.310 usuarios. De este número de pacientes, $40,2 \%$ han recibido de una a cuatro consultas durante su proceso; $24,6 \%$, de cinco a ocho consultas; y $22 \%$, de nueve a doce consultas. El resto, $13,2 \%$, ha recibido desde trece hasta más de veinte consultas en su intervención psicológica. En la mayoría de los casos, los terapeutas atienden a cada usuario una vez por semana. El tiempo de intervención es variable, así como el número de altas, las cuales se dan una vez alcanzados los objetivos terapéuticos.

Gran parte de los usuarios no llega a finalizar su proceso e interrumpen la terapia cuando alcanza parcialmente los objetivos trazados o experimenta una notable mejoría respecto a su sintomatología inicial o su motivo de consulta. De igual forma, es considerable el número de bajas o abandonos de la terapia. No obstante, es elevada la actividad profesional que desarrollan los terapeutas, lo cual contribuye en su formación pedagógica y se logran los objetivos de proyección social de la universidad (Martorell, Carrasco y Ortiz, 2009). De igual forma, Labrador, Estupiñá y García Vera (2010) encontraron que el 52,45 \% había obtenido alta terapéutica; $4,21 \%$ baja terapéutica, y $24,3 \%$ había abandonado el tratamiento. El $83 \%$ tuvo cuatro o menos sesiones de evaluación; y el 75,3\% de los sujetos recibió 18 o menos sesiones de tratamiento. El $24,3 \%$ de los pacientes abandonó el tratamiento antes de su término. El 75,5\% de los pacientes recibió trece sesiones de terapia o menos. Un $68,34 \%$ habían finalizado el tratamiento como altas; y $31,66 \%$, como abandonos.

\section{Conclusiones}

A partir del desarrollo de la presente investigación se puede concluir que la experiencia que ha logrado la Utec a través del modelo de la clínica de atención psicológica, con base a sus buenas prácticas, puede ser un referente para instituciones de educación superior que posean los servicios de clínica psicológica, mediante la articulación de la investigación y la proyección social.

Se ha logrado identificar los niveles de satisfacción y calidad en el servicio de atención psicológica que se brinda en la Clínica Psicológica Utec, referido a usuarios y pacientes y a estudiantes terapeutas en formación.

Se ha logrado identificar, a través de la revisión de diferentes modelos y experiencias de otras instituciones de educación superior, a partir de la experiencia de universidades 
españolas, que los servicios de atención psicológica se integran con las características de los terapeutas, el modelo de atención que adopte la institución y las características de los usuarios.

La Clínica de Psicología de la Utec ha logrado desarrollar los procesos de formación pedagógica en sus estudiantes, 0 terapeutas en formación, ha logrado proyectar a la sociedad y palear las necesidades de salud mental de la población salvadoreña.

Se ha encontrado, como algo de mucha importancia, que los usuarios y terapeutas de la Clínica de Psicología de la Utec muestran un nivel óptimo de satisfacción con el servicio que reciben, y ambos sectores consideran que recomendarían los servicios de la clínica psicológica a un amigo o conocido que lo requieran.

Los usuarios de la Clínica de Psicología se muestran muy satisfechos con el servicio recibido y manifiestan que a través de esta han encontrado lo que buscaban; de igual forma, recomendarían sus servicios.

Los profesionales de la especialidad Clínica de la Psicología de la Utec consideran, en su mayoría, que el modelo de formación y aplicación terapéutica que utiliza la clínica es un modelo ecléctico. Asimismo, son de la opinión de que los terapeutas en formación deben desarrollar más y mejores conocimientos y herramientas para brindar un servicio de calidad.

La formación teórica está basada en el aprendizaje cognitivo conductual. El modelo no está claramente definido, pero está basado en competencias que se van dosificando; y estas se van poniendo en práctica.

La Clínica de Psicología ha logrado fortalecer la formación práctica de los estudiantes de la carrera, a través de una formación teórica y aplicación en su centro de prácticas.

La Clínica de Psicología se ha logrado posicionar en diversos sectores, en los ámbitos de instituciones gubernamentales y de servicio, con la atención que brinda en forma gratuita y de calidad a segmentos de escasos recursos, que no pueden pagar por los servicios de atención psicológica.
La investigación de las buenas prácticas de la Clínica Psicológica de la Utec ha permitido evidenciar que los estudiantes en formación y los usuarios o pacientes de la Clínica se muestran muy satisfechos con los servicios de orientación psicológica que se les brinda.

Los terapeutas hacen patente su satisfacción con la formación y guía que les brindan los docentes y asesores en el área clínica.

\section{Referencias}

Bados López, A.; García Grau, E. \& Fuste Escolano, A. (2002). "Eficacia y utilidad clínica de la terapia psicológica". Universidad de Barcelona, Revista Internacional de Psicología Clínica y de la Salud. 2. 3. 477-502. Barcelona. España.

Belloch, A.; Sandin, B. \& Ramos, F. (2009). Manual de Psicopatología. 2. ${ }^{a}$ Edición McGraw-Hill, Madrid. España.

Beck, A.T.; Rush, A.J.; Shaw, B.F. \& Emery G. (1979). Terapia Cognitiva de la Depresión. 19. ${ }^{a}$ Edición. Editorial Desclée de Brower, S.A., Bilbao. España.

Díaz Curiel, J. (2001). "Revisión de tratamientos psicoterapéuticos en pacientes con trastornos bordeline de personalidad". Revista de la Asociación Española de Neuropsiquiatría. 21, 78, 8, 51-70.

División 12, Asociación Americana de Psicología, APA. "Trastornos psicológicos y problemas de conducta. Sociedad de Psicología Clínica". Recuperado: http:// www.div12.org/psychological-treatments/disorders/

Echeburúa, E.; Corral, P., \& Salaberría, K. (2010). "Efectividad de las terapias psicológicas: un análisis de la realidad actual". Revista de Psicopatología y Psicología Clínica. 15, 2, 85-89.

Gabinete de Psicología. "Algunos enfoques en psicoterapia psicológica", Madrid, $2014 . \quad$ Recuperado: gabinetedepsicología.com/algunos-enfoques-enpsicotrapia-psicologica-psicologos-madrid-tres-cantos Hernández, R.; Fernández, C., \& Baptista, P. (2006). Metodología de la investigación. McGraw-Hill, México. Labrador, F.J.; Estupiñá, F.J. \& García Vera, M.P. (2010) "Demanda de atención psicológica en la práctica clínica: tratamientos y resultados". Universidad Complutense de Madrid. Psicothema. 22. 4. 619-626. 
Makrino, N.; Scharager, J. \& Molina, L. (2005). "Situación actual de una muestra de psicólogos egresados de la Pontificia Universidad Católica de Chile". Psykhe. $14,1,69-77$

Martorell, J.L.; Carrasco Ortiz, M.Á. (2009). "La atención psicológica desde la Universidad Nacional de Educación a Distancia (Uned): El Servicio de Psicología Aplicada (SPA)". Revista Acción Psicológica, 6, 1, 135143. España.

Orellana, L.C. (2006). Historia de la Psicología en El Salvador 1928 -2005. Imprenta Criterio, San Salvador, El Salvador.

Oswaldo Guevara. Blog. Publicación miércoles 30 de enero 2013. "La Historia de la Psicología en El Salvador". Recuperado: http://poga62.blogspot.com/2013/01/lahistoria-de-la-psicologia-en-el.html

Palacios de Flores, C.P.; Castellanos España, R.E.; Escamilla Méndez, M.I. (1989). Tesis: “Propuesta para la creación e implementación de la Unidad de Servicio Psicológico". Presentada en Facultad de Humanidades y Ciencias Naturales, Licenciatura en Psicología, Universidad Tecnológica de El Salvador. San Salvador. El Salvador.

Pineda Romero, R.E.; Guzmán Romero, Y. del C., \& Hernández Guerra, J.L. (2000). Tesis: “Evaluación de los servicios de la Clínica Psicológica de la Universidad Tecnológica. Propuesta para un manual para fortalecer la cobertura de servicios". Presentada en Facultad de Humanidades y Ciencias Naturales, Licenciatura en Psicología, Universidad Tecnológica de El Salvador. San Salvador. El Salvador.

Política Nacional de Salud Mental (2011). Ministerio de Salud. Diagramación e impresión: MKT Plus El Salvador. San Salvador.

Política Nacional de Salud Mental, El Salvador, 2008. http:// www.salud.gob.sv/servicios/descargas/documentos/ Documentación-Institucional/Sistema-Nacional-deSalud/Política-Nacional-de-Salud-Mental/

Políticas, Planes y Programas de Salud Mental (2006). Editores Médicos, S.A., Edimsa, Organización Mundial de la Salud, España.
Portillo, N. (2006). "Antecedentes, desarrollo y aplicaciones temprana de la Psicología en El Salvador (1850-1950). Revista de Historia de la Psicología, 27, 1, 2006, 7-68, publicado por la UCA y el Iudop. San Salvador. El Salvador.

Resultados de la Información Estadística de Instituciones de Educación Superior 2012 (2013). Dirección Nacional de Educación Superior. Ministerio de Educación, República de El Salvador. San Salvador. El Salvador.

Ruiz Sánchez, J.J.; Cano Sánchez, J.J. "Las psicoterapias, introducción a las orientaciones psicoterapéuticas para profesionales sanitarios". Psicología On Line. 2013. Recuperado: www.psicología-online.com/ EsMubeda/libros/ProfSanitarios/profesionales3htm

Sosa, A., y Guzmán Mena, J.A. (1992) Tesis: "Implementación de la Clínica de Orientación Psicológica de la Universidad Tecnológica a través de las prácticas realizadas por los estudiantes egresados de esa orientación". Presentada en la Facultad de Humanidades y Ciencias Naturales, Licenciatura en Psicología, Universidad Tecnológica de El Salvador. San Salvador. El Salvador.

Theorethikos, revista electrónica de la Universidad Francisco Gavidia. Monografía: "El pensamiento de Ignacio Martín-Baró y su aporte a la psicología salvadoreña". (2000) Recuperado: http://www.ufg.edu.sv /ufg/ theorethikos/enero20/monografia1.html

Universidad Centroamericana José Simeón Cañas. UCA. Documento. "Cronología de la violencia, Reporte de la Comisión de la Verdad para El Salvador". Recuperado: http://www.uca.edu.sv/martires/ignaciomartinbaro.htm Universidad José Matías Delgado. Documento publicado: "Nacimiento y Formación de la profesión de Psicología". Recuperado: http://webquery.ujmd. edu.sv/siab/bvirtual/Fulltext/ADMR0000514/ Capitulo\%201.pdf

Vásquez López, L. (2013) Recopilación de Leyes Laborales, Código de Salud. 16. ${ }^{a}$ Edición, Editorial Lis. San Salvador. El Salvador. 